\title{
Analytical inverter chain's delay and its variation model for sub-threshold circuits
}

\author{
Jingjing Guo, Jizhe Zhu, Min Wang, Jianxin Nie, Xinning Liu, \\ Wei Ge, and Jun Yang ${ }^{\text {a) }}$ \\ National ASIC System Engineering Technology Research Center, \\ Southeast University, Nanjing 210096, P.R.C
}

a)dragon@seu.edu.cn

\begin{abstract}
Sub-threshold circuit is a promising circuit design style for IoT application. This paper concentrated on the delay model based on the transient current model in the sub-threshold region. In order to deduce the path delay model, two ways are adopted, which are the coupling capacitance equivalence and the output waveform equivalence. The distribution of path delays is rigidly proven to be lognormal distribution in the sub-threshold region. Considering different supply voltages, cell driven strengths and load capacitances, the proposed model is also validated by Monte Carlo Spice simulation under SMIC $40 \mathrm{~nm}$ CMOS process. Experiments show that proposed model agrees with $\mathrm{MC}$ simulation results with error $0.448 \%$ under the condition of $0.4 \mathrm{~V}$ and $99.7 \%$ probability, which proves the feasibility of the model.
\end{abstract}

Keywords: sub-threshold circuit, lognormal distribution, delay model, variation analysis

Classification: Integrated circuits

\section{References}

[1] Z. Sheng, et al.: "Recent advances in industrial wireless sensor networks toward efficient management in IoT," IEEE Access 3 (2015) 622 (DOI: 10. 1109/ACCESS.2015.2435000).

[2] M. S. Ghaleb, et al:: "Mobility management for IoT: A survey," EURASIP J. Wirel. Commun. Netw. 2016 (2016) 1 (DOI: 10.1186/s13638-016-0659-4).

[3] N. Bari, et al.: "Internet of things as a methodological concept," 2013 Fourth International Conference on Computing for Geospatial Research and Application (COM. Geo) (2013) 48 (DOI: 10.1109/COMGEO.2013.8).

[4] A. Wang, et al.: Sub-Threshold Design for Ultra Low-Power Systems (Springer, 2006).

[5] A. Burdett: "Ultra-low-power wireless systems: Energy-efficient radios for the internet of things," IEEE Solid-State Circuits Mag. 7 (2015) 18 (DOI: 10.1109/ MSSC.2015.2417095).

[6] Y. Tsividis and C. McAndrew: Operation and Modeling of the MOS Transistor (New York, 2011) 3rd ed.

[7] B. Zhai, et al:: "Analysis and mitigation of variability in subthreshold design," Proc. ISLPED (2005) 20 (DOI: 10.1145/1077603.1077610).

[8] M. Orshansky, et al:: Design for Manufacturability and Statistical Design 
(Springer, New York, 2008).

[9] S. Keller, et al:: "A compact transregional model for digital CMOS circuits operating near threshold," IEEE Trans. Very Large Scale Integr. (VLSI) Syst. 22 (2014) 2041 (DOI: 10.1109/TVLSI.2013.2282316).

[10] J. R. Tolbert and S. Mukhopadhyay: "Accurate buffer modeling with slew propagation in subthreshold circuits," Proc. Int. Symp. Qual. Electron. Des. (2009) 91 (DOI: 10.1109/ISQED.2009.4810275).

[11] P. Corsonello, et al:: "Over/undershooting effects in accurate buffer delay model for subthreshold domain," IEEE Trans. Circuits Syst. I, Reg. Papers 61 (2014) 1456 (DOI: 10.1109/TCSI.2013.2285691).

[12] N. Lotze, et al.: "Timing modeling for digital sub-threshold circuits," Proc. Design, Automation and Test in Europe Conf. Exhibition (2010) 299 (DOI: 10. 1109/DATE.2010.5457192).

[13] L. F. Fenton: "The sum of lognormal probability distributions in scatter transmission systems," IRE Trans. Commun. Syst. 8 (1960) 57 (DOI: 10.1109/ TCOM.1960.1097606).

[14] N. A. Marlow: "A normal limit theorem for power sums of independent random variables,” Bell Syst. Tech. J. 46 (1967) 2081 (DOI: 10.1002/j.15387305.1967.tb04244.x).

[15] B. Zhai, et al:: "Analysis and mitigation of variability in subthreshold design," Proc. Int. Symp. Low Power Electron. Design (2005) 20 (DOI: 10.1109/LPE. 2005.195479).

\section{Introduction}

The Internet of things (IoT), which is also called machine to machine communication (M2M), has emerged as a new killer application in which billions of computers and sensor devices are interconnected with each other, enabling an autonomous exchange of information [1, 2, 3]. Low power and ultra-low power dissipation are important while enabling techniques for IoT devices. Due to the square relationship, the lower voltage is, the lower energy dissipation will be. Subthreshold circuit [4] is a promising method for IoT, which operates at a supply voltage less than the transistor threshold voltage. Some specific applications such as wireless sensor devices that require extremely ultra-low power are all operated at sub-threshold voltage [5].

In view of transistor operation mechanisms, MOSFET changes from strong inversion to weak inversion with decreasing voltage, and its primary current also changes from drift to diffusion current [6]. Therefore, the process variation has different influences on the current and delay between super- and sub-threshold [7]. Global and local variations are the two kinds of process variations [8]. Global variation affects all devices in the same way and is the main source of variation in super-threshold. The delay distribution of super-threshold follows normal distribution, which is convenient to calculate. However, local variation influences in different ways even in the same chip and becomes increasingly significant in the sub-threshold. The delay distribution of sub-threshold follows lognormal distribution, which is much harder to analyze.

In order to understand the local variation more, many researches on path delay model under sub-threshold have been studied $[9,10,11]$. In [9], the Ion current 
model is used, and the unit delay model is obtained according to a fitting parameter. Therefore, the unit delay model does not consider the real input waveform and different loads, which only give the result error of unit delay under FO4 load. Besides, the path delay is sum of unit delay with FO4 load, so it is not very reasonable for different loads. For the path delay distribution model, although lognormal distribution is proposed, the computation of the path delay variance should not be the sum of the every unit variation, and it should be obtained according to lognormal theory. The Ids current model is adopted in [10, 11], which considered the transient current with the changing of Vgs and Vds. The delay model is accurate enough especially in [11]. However, there is still a lack of the path delay distribution modeling with process parameter variation.

In this paper, an analytical method involving the path delay distribution is proposed. The main contributions of this work are as followed. The exponential relationship between delay and threshold voltage is demonstrated in detail in Section 2, which has not been rigidly proved so far to our knowledge. Section 3 introduces the lognormal distribution theory, and path delay distribution is obtained under different voltages as well.

\section{Delay model}

Some analytical delay estimation techniques have been developed recently in $[10,11]$. The transient current of NMOS and PMOS can be accurately approximated, and the unit and path delay are well estimated. However, none of path delay model take $V_{t h}$ variation into account.

\subsection{Sub-threshold current}

The sub-threshold drain-source current [11] of NMOS is given by

$$
I_{d s n}=I_{0 n} \frac{W_{n}}{L} \cdot \exp \left(\frac{V_{g s}-V_{t h n 0}}{n_{n} V_{T}}\right) \cdot \exp \left(\frac{\lambda_{n} V_{d s}}{n_{n} V_{T}}\right) \cdot\left(1-e^{-\frac{V_{d s}}{V_{T}}}\right)
$$

where

$$
\begin{gathered}
I_{0 n}=\mu_{n} C_{o x}\left(n_{n}-1\right) V_{T}^{2} \\
V_{T}=k T / q .
\end{gathered}
$$

$\lambda_{n}$ is the DIBL (Drain Induced Barrier Lowering) coefficient, $V_{T}$ is the thermal voltage, $V_{t h n}$ is the threshold voltage at zero bias voltage and $n_{n}$ is the subthreshold slope factor. A variable $M_{n}$ is defined and expressed by

$$
M_{n}=I_{0 n} \frac{W_{n}}{L} \exp \left(-\frac{V_{t h n 0}}{n_{n} V_{T}}\right) .
$$

Similarly, the drain-source current of PMOS can be obtained.

The sub-threshold current with changing input and output voltage is shown by the arrows in Fig. 1. S. Keller, et al. [9] build the delay model based on the on state current Ion, which is marked by red point. Obviously, Ion is greater than the actual current and cannot accurately represent the entire current, so the delay model is built based on a fitting parameter with FO4 load in [9]. However, in this paper, the transient current is studied based on (1), which reflect the relationship with $V_{g s}$ and 


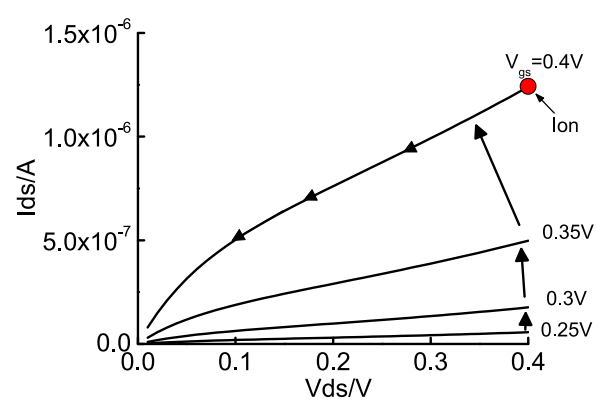

Fig. 1. Transient current of NMOS.

$V_{d s}$ respectively, and the ramp input is also considered, so the delay model is built without any fitting parameters.

Table I. Technology parameters of transistor in SMIC40 nm.

\begin{tabular}{lll}
\hline Parameters & NMOS & PMOS \\
\hline$I_{0}(\mathrm{~A})$ & $7.74 \mathrm{e}-09$ & $2.263 \mathrm{e}-09$ \\
\hline$\lambda$ & 0.13 & 0.16 \\
\hline$V_{t h 0}(\mathrm{~V})$ & 0.4895 & -0.572 \\
\hline $\mathrm{n}$ & 1.89 & 1.92 \\
\hline
\end{tabular}

For SMIC $40 \mathrm{~nm}$ LVT technology at nominal temperature, transistor parameters are listed in Table I, where $L=40 \mathrm{~nm}$ and $W_{P}=W_{N}=120 \mathrm{~nm}$. The comparisons of the transient current model with Spice simulation are shown in Fig. 2, where (a) and (b) are the results of NMOS and PMOS respectively.

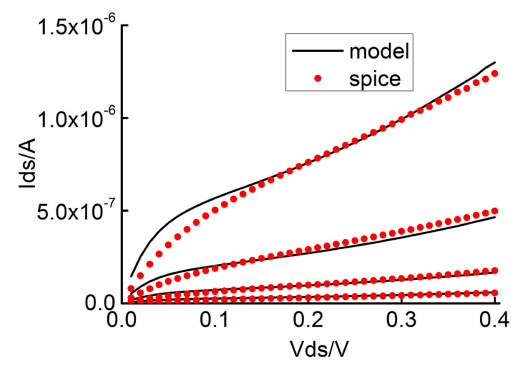

(a) NMOS

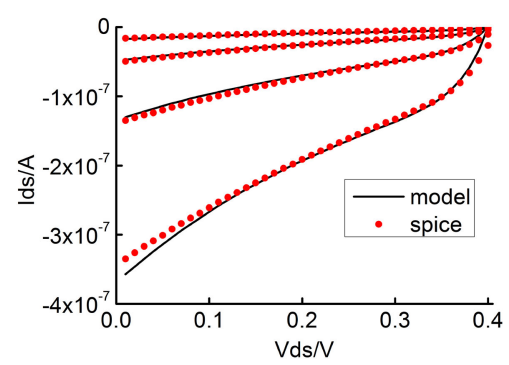

(b) PMOS

Fig. 2. Comparisons of transistor transient current with Spice.

\subsection{Output waveform}

According to papers $[10,11]$, the output waveform can be expressed by piecewise function. In this paper, in order to simplify the computation and keep its accuracy, two ways are adopted in the modeling.

\subsubsection{Gate-drain coupling capacitance equivalence}

J. R. Tolbert et al. [10] do not consider coupling capacitance, and P. Corsonello et al. [11] have a complicated output waveform expression when taking coupling 
capacitance into consideration, which results in complexity of the following delay model. Therefore, Miller equivalent method is adopted to simplify the complexity in this paper.
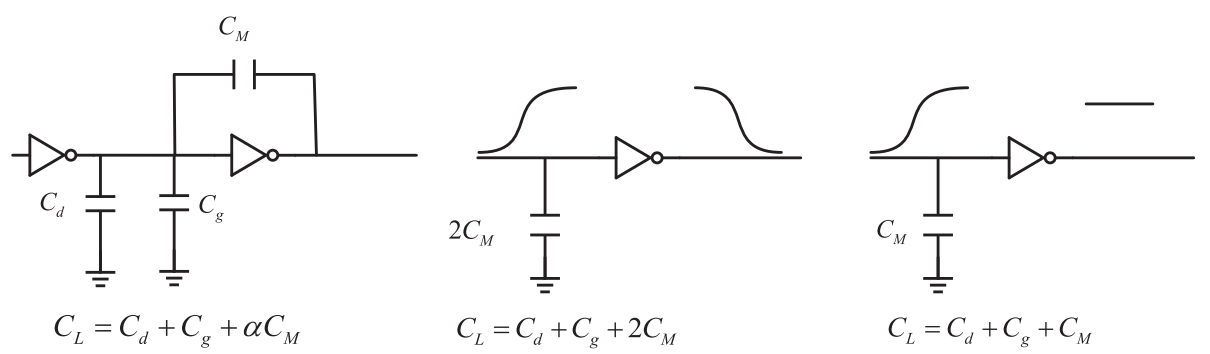

Fig. 3. Coupling capacitance equivalence.

Fig. 3 shows that coupling capacitance $\left(C_{M}\right)$ exists between gate and drain of MOSFET. When the input and output waveform change in different directions, $2 C_{M}$ is equal to the gate port, while $C_{M}$ is equivalent capacitance with output unchanged. Moreover, in order to further simplify the capacitance, averaged $1.5 C_{M}$ is used in the following delay computation.

Therefore, the equation of falling output waveform is expressed as

$$
V_{\text {out }}= \begin{cases}\frac{-n_{n} V_{T}}{\lambda_{n}} \cdot \ln \left(\frac{M_{n} \lambda_{n} \tau}{V_{d d} C_{L}}\left(e^{\frac{V_{d d}}{n_{n} V_{T} t}}-1\right)+e^{-\frac{\lambda_{n} V_{d d}}{n_{n} V_{T}}}\right) & 0<\mathrm{t}<\tau \\ \frac{-n_{n} V_{T}}{\lambda_{n}} \cdot \ln \left(e^{-\frac{\lambda_{n} V_{\text {out }}-\tau}{n_{n} T_{T}}}+\frac{M_{n} \lambda_{n} e^{\frac{V_{d d} V_{T}}{n_{n} V_{T}}}}{C_{L} n_{n} V_{T}}(t-\tau)\right) & \tau<\mathrm{t}<\mathrm{t}_{3} 3 \mathrm{~V}_{\mathrm{T}} \\ V_{T} \cdot \ln \left(1+\left(e^{3}-1\right) \cdot e^{-\frac{M_{n} V_{d d} V_{n} V_{n} V_{T}}{V_{T} C_{L}}\left(t-t_{-3 V T}\right)}\right) & \mathrm{t}>\mathrm{t}_{-} 3 \mathrm{~V}_{\mathrm{T}}\end{cases}
$$

In a similar way, the result of the rising output waveform is given by

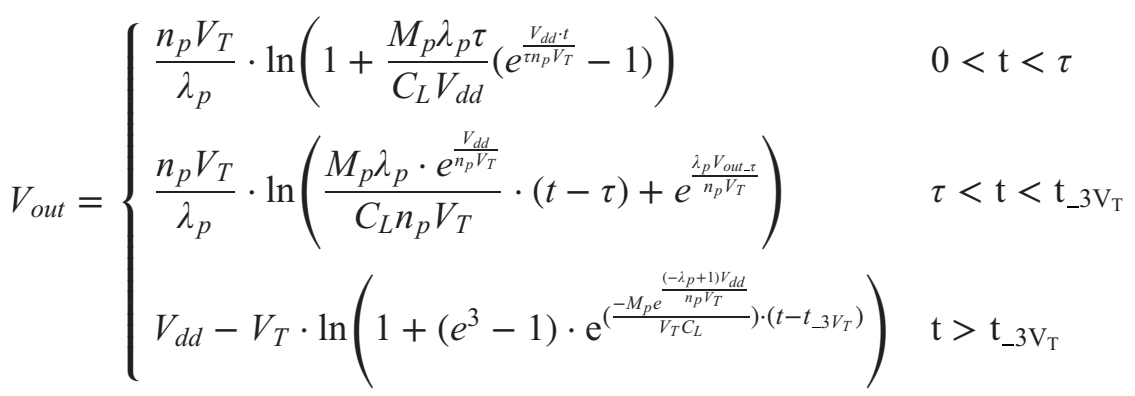

\subsubsection{Output waveform equivalence}

There exist some papers introducing how to approximate output waveform. P. Corsonello et al. [11] divide output waveform into four linear parts and two of them are ramp, making the delay model complicated. N. Lotze et al. [12] deal a formula that fits well in the range of $V_{d d} / 2$ to 0 for the fall transition and $V_{d d} / 2$ to $V_{d d}$ for the rise transition. The reason is that the last part for every transition has a much more significant effect on output voltage.

Combining the two minds, an approximate method is proposed and shown in Fig. 4. A line is used to describe the last part of every transition. Take the fall transition as an example, $t_{1}$ and $t_{2}$ are the corresponding points of output voltage 


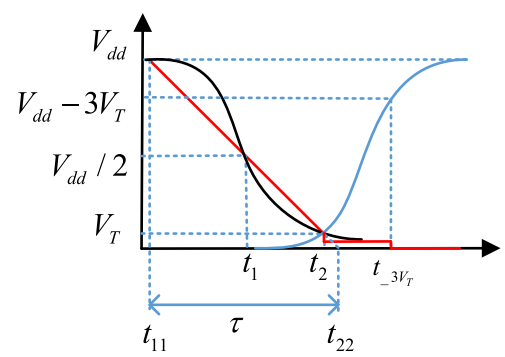

Fig. 4. Linear approximation of output waveform.

achieved $V_{d d} / 2$ and $V_{T}$ respectively. Then the line is extrapolated to $V_{d d}$ and 0 , and the corresponding points are $t_{11}$ and $t_{22}$. The difference between $t_{11}$ and $t_{22}$ is considered as the transition time of the next stage. Between $t_{2}$ and $t_{-3 V_{T}}$, the supply is $V_{d d}-0.3 V_{T}$, and the supply value is based on specific technology. In the last part the voltage is $V_{d d}$ again. This way not only keeps it simple and convenient for next work but also fits the important part and keeps its accuracy.

\subsubsection{Output waveform validation}

Experiment is done to validate the proposed model with different sizes and loads. The circuit structure is shown in Fig. 5(a), where M represents the drive strength. The comparison of the model with Spice is shown in Fig. 5(b), which shows a good agreement between them.

\subsection{Delay model}

\subsubsection{Derivation of delay model}

Fig. 5(b) shows that the majority of second part in (5) are across the point of $V_{d d} / 2$. We consider the schematic in Fig. 6 and derive the delay formula, where $t_{p h l}$ represents the falling delay, $\tau_{i}$ is the transition time of the $i_{t h}$ stage, and $C_{L i}$ is load capacitance.

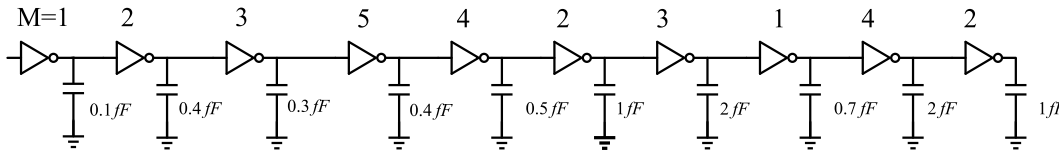

(a) Circuit Structure

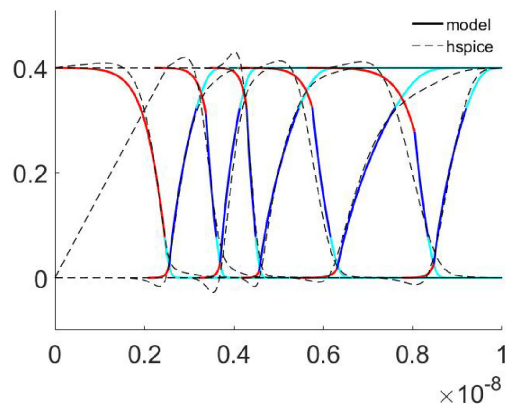

(b) Output Waveform 


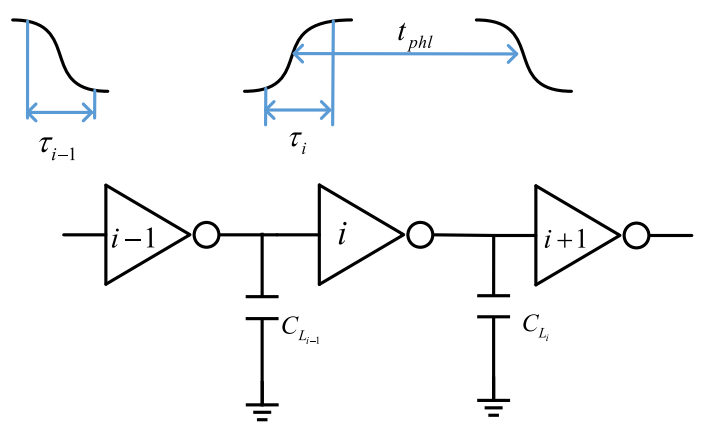

Fig. 6. Schematic of inverter chains used for delay model

$$
\begin{aligned}
t_{p h l}= & \frac{n_{n} V_{T}}{I_{0 n} \lambda_{n}} \frac{C_{L i}}{W_{n} / L} e^{\frac{-V_{d d}+0.3 V_{T}}{n_{n} V_{T}}} \cdot\left(e^{\frac{-\lambda_{n} V_{d d / 2}}{n_{p} T_{T}}}-e^{\frac{-\lambda_{n} V_{d d}}{n_{p} V_{T}}}\right) \cdot \exp \left(\frac{V_{t h n}}{n_{n} V_{T}}\right) \\
& +\left(\frac{1}{2}+\frac{C_{4}}{V_{d d}}\right) \tau_{i} .
\end{aligned}
$$

Equation (10) shows, the delay of the $i_{t h}$ stage inverter includes two parts. The first part is the step delay of $i_{t h}$ stage, and the second part is the influence of the $(i-1)_{t h}$ stage. And the expression of $\tau_{i}$ is needed to derive.

According to Fig. $4, \tau_{i}$ can be expressed by $t_{1}$ and $t_{2}$ :

$$
\tau_{i}=\left(t_{2}-t_{1}\right) \cdot\left(\frac{V_{d d}}{V_{d d} / 2-V_{T}}\right) .
$$

The difference between $t_{2}$ and $t_{1}$ is described as

$$
t_{2}-t_{1}=\frac{C_{L i-1}}{W_{p} / L} \cdot C_{3} \cdot\left(C_{1}-C_{2} \cdot e^{\frac{-\lambda_{1} V_{d d}}{2 n_{p} V_{T}}}\right) \cdot e^{\frac{-V_{d d}}{n_{p} V_{T}}} \cdot \exp \left(\frac{V_{t h p}}{n_{p} V_{T}}\right)
$$

where $\quad C_{1}=-\ln \left(\frac{e-1}{e^{3}-1}\right)+\frac{n_{p}}{\lambda_{p}} \exp \left(\frac{0.3-3 \lambda_{p}}{n_{p}}\right), \quad C_{2}=\frac{n_{p}}{\lambda_{p}} \exp \left(\frac{0.3}{n_{p}}\right), \quad C_{3}=\frac{V_{T}}{I_{0 p}}, \quad C_{4}=$ $\left(-1-n_{n} \exp \left(\frac{-0.7}{n_{n}}\right)\right) V_{T}$, and can be obtained by technology parameters.

So the expression of $t_{p h l}$ is

$$
t_{p h l}=f_{1}\left(V_{d d}, \frac{C_{L_{i}}}{W_{n} / L}\right) \cdot \exp \left(\frac{V_{t h n}}{n_{n} V_{T}}\right)+f_{2}\left(V_{d d}, \frac{C_{L_{i-1}}}{W_{p} / L}\right) \cdot \exp \left(\frac{V_{t h p}}{n_{p} V_{T}}\right)
$$

where

$$
\begin{gathered}
f_{1}\left(V_{d d}, \frac{C_{L_{i}}}{W_{n} / L}\right)=\frac{n_{n} V_{T}}{I_{0 n} \lambda_{n}} \frac{C_{L i}}{W_{n} / L} e^{\frac{-V_{d h+0.3 V_{T}}}{n_{n} V_{T}}} \cdot\left(e^{\frac{-\lambda_{n} V_{d d} / 2}{n_{p} V_{T}}}-e^{\frac{-\lambda_{n} V_{d d}}{n_{p} V_{T}}}\right) \\
f_{2}\left(V_{d d}, \frac{C_{L_{i-1}}}{W_{n} / L}\right)=\left(\frac{1}{2}+\frac{C_{4}}{V_{d d}}\right) \cdot \tau_{i} \cdot e^{\frac{-V_{n p} V_{p}}{n_{p} V_{T}}} .
\end{gathered}
$$

Similarly, the expression of $t_{p l h}$ can be obtained. And the delay of each stage has an exponential relationship with current and previous stage threshold voltage.

\subsubsection{Delay model validation}

The comparison of the delay model with Spice simulation is shown in Fig. 7. Besides, the result is also explored at $0.35 \mathrm{~V}$ and $0.3 \mathrm{~V}$, which shows that they have a good agreement with each other, with errors are $1.42 \%, 4.03 \%$, and $3.39 \%$, respectively. 


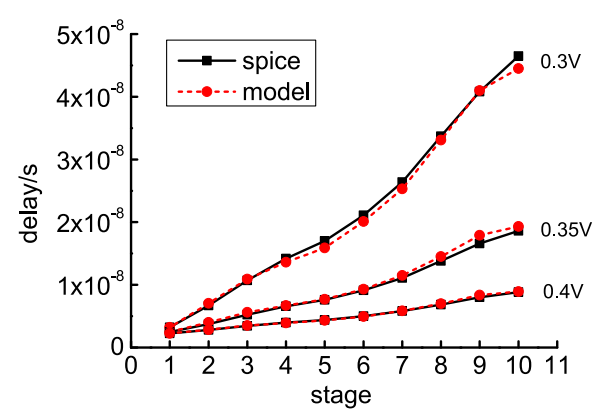

Fig. 7. Every stage delay of the arbitrary size inverter chains at different voltages.

\section{Delay distribution model for sub-threshold}

For discussion on the delay distribution, the concepts of probability density function (PDF) and cumulative density function (CDF) are introduced firstly.

\subsection{Lognormal distribution}

If a Random Variable (RV) X obeys a standard normal distribution, $Y=\exp (x)$ obeys lognormal distribution. Its PDF and CDF are

$$
\begin{gathered}
\ln N\left(y ; \mu, \sigma^{2}\right)=\frac{1}{y \sqrt{2 \pi} \sigma} \exp \left(-\frac{(\ln y-\mu)^{2}}{2 \sigma^{2}}\right) \\
\int_{0}^{y} \ln N\left(y ; \mu, \sigma^{2}\right) d y=\phi\left(\frac{\ln y-\mu}{\sigma}\right),
\end{gathered}
$$

where $\phi$ is the CDF of standard normal distribution.

If $Y_{j}$ is an independent lognormal distribution variable, $Z=\sum_{j=1}^{N} Y_{j}$ also obeys lognormal distribution. The variance and mean of $\mathrm{Z}$ are shown as following $[13,14]$ :

$$
\begin{gathered}
\sigma_{z}^{2}=\ln \left(\frac{\sum e^{2 \mu_{j}+\sigma_{j}^{2}}\left(e^{\sigma_{j}^{2}}-1\right)}{\left(\sum e^{\mu_{j}+\sigma_{j}^{2} / 2}\right)^{2}}+1\right) \\
\mu_{z}=\ln \left(\sum e^{\mu_{j}+\sigma_{j}^{2} / 2}\right)-\frac{\sigma_{z}^{2}}{2} .
\end{gathered}
$$

\subsection{Path delay distribution in sub-threshold}

Process variations include variations of threshold voltage, effective channel length, doping concentration, and more parameters. Since threshold voltage variation is a dominant factor affecting the delay in the subthreshold region [15], the following model only considers the variations of $V_{t h}$.

The delay of the $i_{t h}$ stage is expressed as

$$
t_{p h l}(i)=f_{1}(i) \cdot \exp \left(\frac{V_{t h n 0}}{n_{n} V_{T}}\right)+f_{2}(i) \cdot \exp \left(\frac{V_{t h p 0}}{n_{p} V_{T}}\right) .
$$

And the delay of the $(i+1)_{t h}$ stage is

$$
t_{p l h}(i+1)=f_{3}(i+1) \exp \left(\frac{V_{t h p 0}}{n_{p} V_{T}}\right)+f_{4}(i+1) \exp \left(\frac{V_{t h n 0}}{n_{n} V_{T}}\right) .
$$


So the path delay can be expressed as

$$
t_{p}=\sum\left\{\begin{array}{l}
{\left[f_{1}(i)+f_{4}(i+1)\right] \cdot \exp \left(\frac{V_{t h n}}{n_{n} V_{T}}\right)} \\
+\left[f_{3}(i+1)+f_{2}(i+2)\right] \cdot \exp \left(\frac{V_{t h p 0}}{n_{p} V_{T}}\right)
\end{array}\right\} \quad(i=1,3,5 \ldots) .
$$

Based on the transistor BSIM4 model, variation of $V_{\text {th }}$ abides by standard normal distribution. We have the following expression:

$$
V_{t h n 0} \sim N\left(\mu_{n}, \sigma_{n}{ }^{2}\right)
$$

where $u_{n}$ is the mean and $\sigma_{n 0}^{2}$ is the variance of minimal width transistor ( $W_{N 0}=$ $120 \mathrm{~nm}$ and $L=40 \mathrm{~nm}$ )

$$
\sigma_{n}^{2}=\left(\frac{\sigma_{n 0}}{\sqrt{W_{N} / W_{N 0}}}\right)^{2}
$$

According to the property of standard normal distribution, we have

$$
\frac{V_{t h n 0}}{n_{n} V_{T}} \sim N\left(\frac{\mu_{n}}{n_{n} V_{T}},\left(\frac{\sigma_{n}}{n_{n} V_{T}}\right)^{2}\right) .
$$

Based on the definition of lognormal distribution, $\exp \left(V_{t h n} / n_{n} / V_{T}\right)$ follows lognormal distribution

$$
\exp \left(\frac{V_{t h n 0}}{n_{n} V_{T}}\right) \sim \ln N\left(\frac{\mu_{n}}{n_{n} V_{T}},\left(\frac{\sigma_{n}}{n_{n} V_{T}}\right)^{2}\right),
$$

and

$$
\begin{aligned}
& {\left[f_{1}(i)+f_{4}(i+1)\right] \cdot \exp \left(\frac{V_{t h n 0}}{n_{n} V_{T}}\right)} \\
& \sim \ln N\left(\frac{\mu_{n}}{n_{n} V_{T}}+\ln \left[f_{1}(i)+f_{4}(i+1)\right],\left(\frac{\sigma_{n}}{n_{n} V_{T}}\right)^{2}\right) \\
& {\left[f_{3}(i+1)+f_{2}(i+2)\right] \cdot \exp \left(\frac{V_{t h p 0}}{n_{p} V_{T}}\right)} \\
& \sim \ln N\left(\frac{\mu_{p}}{n_{p} V_{T}}+\ln \left[f_{3}(i+1)+f_{2}(i+2)\right],\left(\frac{\sigma_{p}}{n_{p} V_{T}}\right)^{2}\right),
\end{aligned}
$$

where $f_{1}, f_{2}, f_{3}$ and $f_{4}$ can be obtained based on the size and load of each stage. And according to (15) (16), the mean and variance of path delay can be obtained.

Fig. 8 shows the proposed model and spice match very well at $0.4 \mathrm{~V}$. Furthermore, the delay distributions at $0.35 \mathrm{~V}$ and $0.3 \mathrm{~V}$ are explored. The error of path delay distribution is shown in Fig. 9, which is $0.906 \%(1.78 \%, 0.448 \%)$ when the probability is $0.3 \%(50 \%, 99.7 \%)$. And the error of other voltages are listed in Table II.

From the view of the principle and method of delay model, path delay error and distribution error, comparisons with previous works $[9,10,11]$ are concluded and shown in Table II. [10] and [11] study the delay model in detail and not do the delay distribution model. [9] study the delay and delay distribution model, but the current model adopt on state current, so delay model based on fitting parameters, 


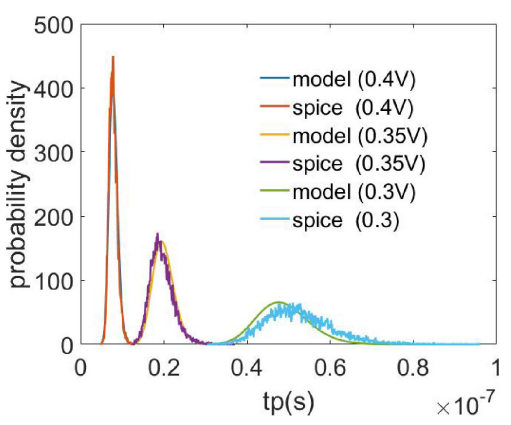

(a) pdf

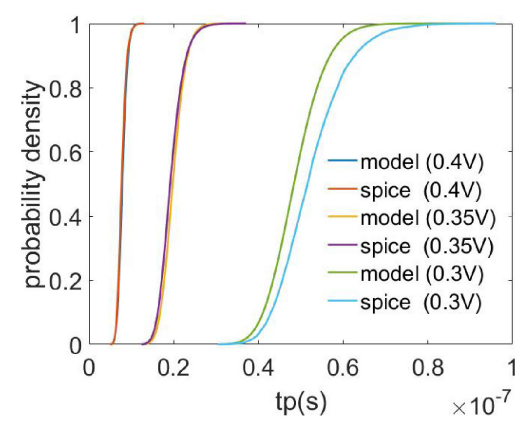

(b) $\mathrm{cdf}$

Fig. 8. The comparison of path delay distribution of model with $10 \mathrm{~K}$ $\mathrm{MC}$ Spice simulation.

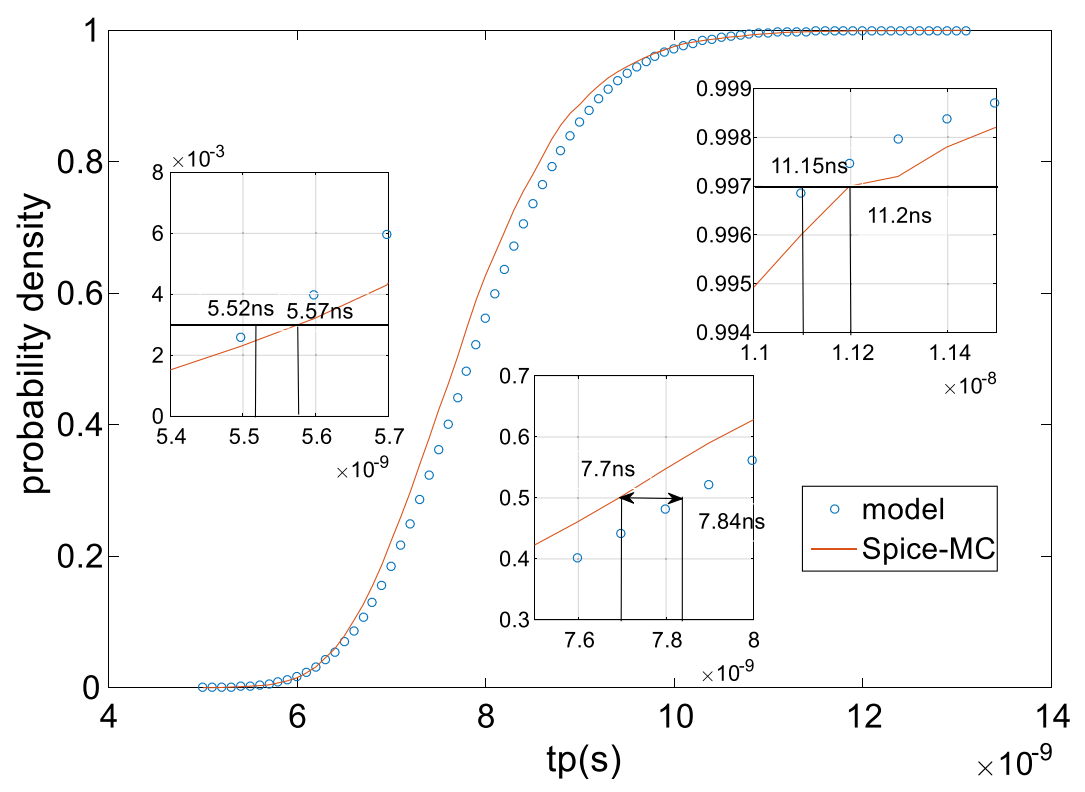

Fig. 9. Path delay distribution error.

and besides, the mean and variation of path delay is not based on lognormal theory. In this paper, the transient current model is used, so no fitting parameters such as $K$ in [9] is incorporated in the delay model. For path delay model, Miller equivalence and output waveform simplicity are adopted, which have two sides. The advantage is making the model much simpler to compute the following delay distribution. And the disadvantage is bringing a side effect on the accuracy compared with [11], yet accurate enough for the following computation of path delay distribution. Besides, path delay distribution is proposed in this paper based on lognormal theory and the comparison results with [9] is shown in Table II, which is more accurate than [9]. 
Table II. Comparison with previous works

\begin{tabular}{|c|c|c|c|c|c|c|c|}
\hline & & [9] & {$[10]$} & {$[11]$} & \multicolumn{3}{|c|}{ This work } \\
\hline \multirow{8}{*}{$\begin{array}{c}\text { Delay } \\
\text { model } \\
\text { Principle } \\
\text { and } \\
\text { method }\end{array}$} & Ids Model & $\begin{array}{c}I_{d s}= \\
k \cdot I_{o n} \\
\mathrm{k}: \text { fitting } \\
\text { para. }\end{array}$ & \multicolumn{5}{|c|}{$\begin{array}{c}I d s=f\left(V_{g s}, V_{d s}\right) \\
\text { no fitting } \\
\text { parameters }\end{array}$} \\
\hline & Supply & \multicolumn{6}{|c|}{ interval } \\
\hline & Temp. & \multicolumn{6}{|c|}{ single } \\
\hline & $\begin{array}{l}\text { Input } \\
\text { Slew }\end{array}$ & Step & \multicolumn{5}{|c|}{$\begin{array}{c}\text { Ramp input } \\
\text { derived by previous stage }\end{array}$} \\
\hline & $\begin{array}{l}\text { Output } \\
\text { Slew }\end{array}$ & $\mathrm{N} / \mathrm{A}$ & $\mathrm{N} / \mathrm{A}$ & $\begin{array}{l}\text { two } \\
\text { ramp } \\
\text { part }\end{array}$ & \multicolumn{3}{|c|}{$\begin{array}{l}\text { one } \\
\text { ramp part }\end{array}$} \\
\hline & load & FO4 & \multicolumn{5}{|c|}{ actual condition } \\
\hline & $\begin{array}{l}\text { Coupling } \\
\text { Cap. }\end{array}$ & No & No & $\begin{array}{c}\text { Yes: } \\
\text { direct } \\
\text { compute }\end{array}$ & \multicolumn{3}{|c|}{$\begin{array}{c}\text { Yes: } \\
\text { miller } \\
\text { equivalence }\end{array}$} \\
\hline & $\begin{array}{l}\text { Path } \\
\text { delay } \\
\text { distri. }\end{array}$ & $\begin{array}{c}\text { lognormal: } \\
\text { u,sigma } \\
\text { not } \\
\text { based on } \\
\text { lognormal } \\
\text { theory }\end{array}$ & No & No & \multicolumn{3}{|c|}{$\begin{array}{c}\text { lognormal: } \\
u, \text { sigma } \\
\text { based on } \\
\text { lognormal } \\
\text { theory }\end{array}$} \\
\hline \multirow{12}{*}{$\begin{array}{l}\text { path } \\
\text { delay } \\
\text { error }\end{array}$} & Stage $\mathrm{Vdd}$ & $0.3 \mathrm{~V}$ & $\mathrm{~N} / \mathrm{A}$ & $0.3 \mathrm{~V}$ & $0.4 \mathrm{~V}$ & $0.35 \mathrm{~V}$ & $0.3 \mathrm{~V}$ \\
\hline & 1 & & $3.2 \%$ & $1.75 \%$ & $0.86 \%$ & $1.23 \%$ & $1.74 \%$ \\
\hline & 2 & $7.8 \%$ & $13 \%$ & $0.24 \%$ & $1.06 \%$ & $6.67 \%$ & $3.05 \%$ \\
\hline & 3 & & $10 \%$ & $0.71 \%$ & $1.45 \%$ & $7.05 \%$ & $5.33 \%$ \\
\hline & 4 & & $13 \%$ & $0.9 \%$ & $1.01 \%$ & $1.82 \%$ & $1.87 \%$ \\
\hline & 5 & & $13 \%$ & $0.27 \%$ & $1.37 \%$ & $0.92 \%$ & $4.23 \%$ \\
\hline & 6 & & $13 \%$ & & $0.80 \%$ & $2.08 \%$ & $6.47 \%$ \\
\hline & 7 & & $15 \%$ & & $0.34 \%$ & $3.60 \%$ & $4.74 \%$ \\
\hline & 8 & & $14 \%$ & & $1.75 \%$ & $5.07 \%$ & $4.17 \%$ \\
\hline & 9 & & $18 \%$ & & $4.62 \%$ & $7.83 \%$ & $1.78 \%$ \\
\hline & 10 & $8.9 \%$ & $15 \%$ & & $0.90 \%$ & $3.76 \%$ & $0.49 \%$ \\
\hline & avg error & & $12.72 \%$ & $0.77 \%$ & $1.42 \%$ & $4.03 \%$ & $3.39 \%$ \\
\hline \multirow{3}{*}{$\begin{array}{l}\text { CDF } \\
\text { error }\end{array}$} & $\mathrm{P}=0.3 \%$ & $43.75 \%$ & & & $0.906 \%$ & $2.95 \%$ & $3.23 \%$ \\
\hline & $P=50 \%$ & $34.28 \%$ & & & $1.78 \%$ & $3.04 \%$ & $6.65 \%$ \\
\hline & $P=99.7 \%$ & $8.5 \%$ & & & $0.448 \%$ & $4.64 \%$ & $18.25 \%$ \\
\hline
\end{tabular}

\section{Conclusions}

In this paper, transient current is used to deduce unit the delay model. In order to simplify the computation process of obtaining the path delay model, two ways are adopted, which are coupling capacitance and output waveform equivalence. And they are explored under different voltages. According to the lognormal distribution theory, the path delay distribution model is obtained, which is consistent to spice $\mathrm{MC}$ simulation. 


\section{Acknowledgments}

This work was supported by National Natural Science Foundation of China (Grant No. 61474022 and 61404028) and National High Technology Research and Development Program 863 (Grant No. 2015AA016601). 